\title{
ПРОВОДНАЯ СВЯЗЬ
}

\section{ОПТИЧЕСКАЯ МАРШРУТИЗАЦИЯ в транспортнои сети IMT-2020/5G}

Е.Богданова, инженер ООО "Т8 НТЦ" / bogdanova@t8.ru

УДК 621.39, DOI: 10.22184/2070-8963.2020.86.1.62.70

Рассматриваются вопросы маршрутизации в транспортной сети IMT-2020/5G, а также программно-аппаратный комплекс оборудования для сегмента Backhaul и требования к нему. С учетом необходимости формирования сквозных соединений в сети 5G предлагается метод построения оптимального маршрута на оптическом уровне с двухпараметрической метрикой и весовыми коэффициентами каждого параметра, которые могут определяться сценарием 5G. В качестве критериев рекомендуются наиболее важные характеристики сети IMT-2020/5G: задержка и оптическое отношение сигнал/шум (OSNR).

\section{ВведенИЕ}

Стандарт IMT-2020/5C описывает построение сети мобильной связи пятого поколения. В отличие от предшествующих технологий, в частности 4G-LTE, 5G влечет за собой изменение не только участка мобильного доступа, но и всей инфраструктуры - от радиочасти до транспортной сети и единой плоскости управления.

Общая архитектура транспортной сети IMT-2020/5C представлена на рис.1 и подробно рассмотрена в статье [1]. Фокус данной работы - оптическая маршрутизация в сегменте Backhaul со сложной топологией (mesh, кольцо, смешанная топология).

В сетях IMT-2020/5G задача транспорта более сложная, чем поддержание достаточной пропускной способности, что успешно решается стеком технологий передачи данных OTN/DWDM. Сервис-провайдеры обязаны обеспечить пропускную способность по требованию и быструю реконфигурацию транспортных

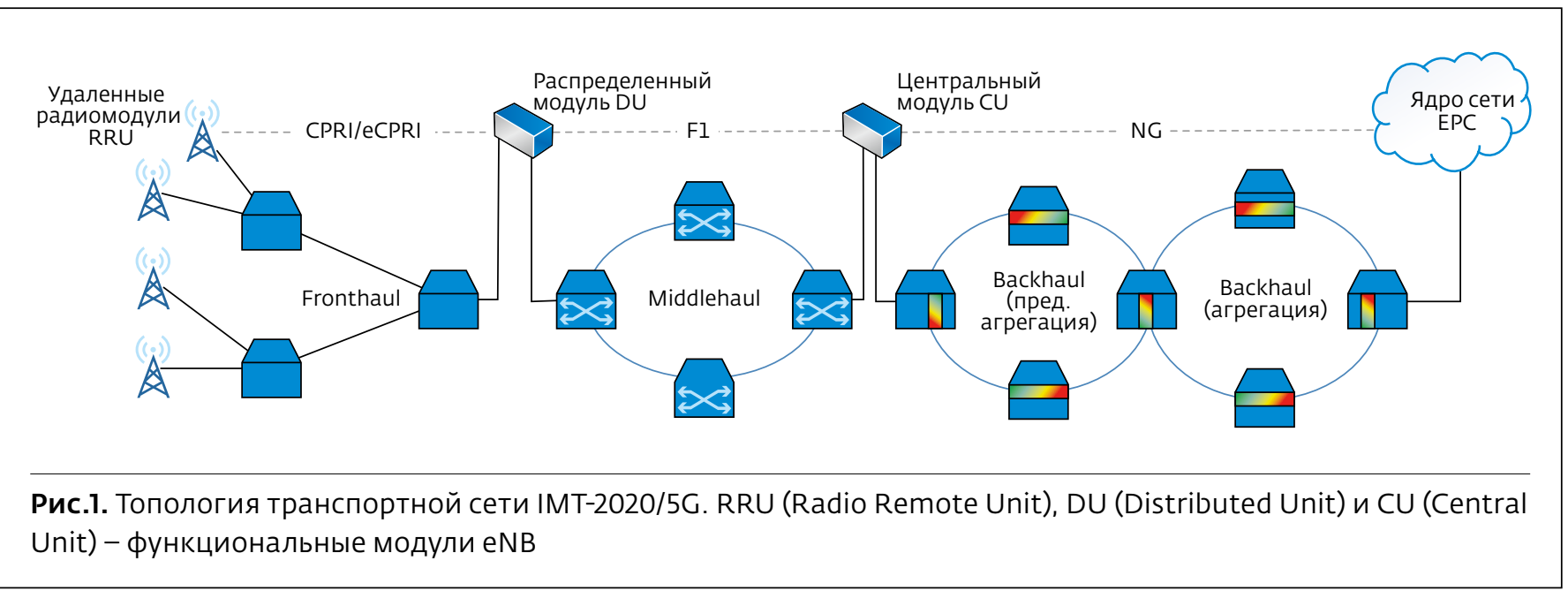


ресурсов с учетом сценария, а производители оборудования - соответствующие механизмы на аппаратном и программном уровнях. В результате абоненту или сервису должен быть предоставлен сквозной туннель по оптимальному сетевому маршруту с учетом качества передачи, задержек и других критичных параметров.

Параметр, который прежде не относился к определяющим при построении маршрута на физическом уровне (L0), но критично важный в сетях 5G - это задержка. При формировании маршрута также необходимо учитывать качество линейного сигнала для надежной безошибочной передачи высокоскоростных сигналов.

В данной статье предлагается решение задачи построения маршрута на оптическом уровне сегмента Backhaul с учетом упомянутых выше параметров: задержки и оптического отношения сигнал/ шум, характеризующего качество сигнала.

\section{ОСОБЕННОСТИ СЕГМЕНТА ВАСКНАUL}

У каждого сегмента своя архитектура, поэтому и требования к транспортному оборудованию различаются. Например, выбор технологии для Fronthaul определяется опцией функционального разделения в RAN (англ. RAN - Radio Access Network). Согласно рекомендациям [3] транспортная сеть Fronthaul должна реализовывать топологию точка-точка (точка-многоточка), а значит, отпадает необходимость маршрутизации. Middlehaul может представлять собой кольцо с максимальным расстоянием между сетевыми элементами до 40 км.

Сегмент Backhaul - классический вариант для оборудования высокоскоростного оптического транспорта - объединяет трафик базовых станций и доставляет его к ядру сети. Backhaul может представлять собой mesh-топологию, оптическое кольцо или гибридную топологию. Протяженность оптического маршрута достигает 450 км согласно [3].

Плоскость передачи данных Backhaul предпочтительно организовывать в виде стека DWDM/OTN, так как эти технологии L0/L1 обеспечивают необходимую пропускную способность в сочетании с высокой надежностью соединений. Однако требования со стороны 5G-сервисов, помимо минимизации задержек, усложняют технологию оптического транспорта необходимостью обеспечить интеллектуальные плоскости контроля и управления. Стандартные процедуры OAM (англ. OAM - Operation, Administration and Maintenance), предусмотренные в OTN, должны быть расширены механизмами GMPLS и SDN. Их назначение - маршрутизация, предоставление пропускной способности и возможности реконфигурации сетевых ресурсов по запросу сервисов 5G.

B сети OTN/DWDM маршрутизация может быть реализована на устройствах L0, в мультиплексорах ROADM (англ. ROADM - Reconfigurable Optical Add/ Drop Multiplexor), на уровень выше (L1), в узлах с OTN кросс-коннектом (транспондерах, OTN-коммутаторах). В первом случае протокол GMPLS работает с отдельными длинами волн, во втором происходит разбор сигнала на электрическом уровне, и клиентский трафик передается в направлении, определяемом протоколом маршрутизации.

Кроме того, необходимую гибкость можно обеспечить благодаря использованию перестраиваемых по длине волны транспондеров с возможностью изменения формата модуляции в зависимости от запрашиваемого конечными сервисами маршрута.

В первых коммерческих проектах по внедрению 5G операторы не планируют модернизировать инфраструктуру Backhaul. По их мнению, сеть, построенная с учетом роста трафика 4G, выдержит нагрузку от редких абонентов 5С. Однако после выхода второй фазы стандарта 3СРP ситуация может измениться.

Модернизация Backhaul может потребоваться операторам при введении URRLC-сценария, который предположительно будет описан в релизе 16 стандарта 3GPP: по некоторым оценкам, суммарная двусторонняя задержка (англ. RTT - Round Trip Time) не должна 
превышать в сети 1 мс. Более реалистичное значение которое приводится в [3] - это 1 мс - требование к RTT между узлами ядра сети.

На сегодняшний день типовыми значениями задержек прохождения трафика через блоки DWDM считаются единицы-десятки наносекунд для обору дования уровня L0 и единицы-десятки микросекунд для транспондеров и блоков кросс-коннекта с OTN коммутацией на уровне L1. Ethernet-маршрутизаторы уровня L2 вносят задержку в десятки-сотни микросекунд, причем без механизмов TSN (англ. TSN - Time Sensitive Networking) значения задержки варьируются в зависимости от загрузки. Наибольший вклад в задержку группового сигнала вносит оптическое волокно, распространение сигнала по которому оценивается в 5 мкс/км.

\section{ПЛОСКОСТИ УПРАВЛЕНИЯ И КОНТРОЛЯ}

Оптическую сеть можно представить в виде трех взаимодействующих уровней - плоскости данных, плоскости контроля и плоскости управления (в некоторых источниках последние два уровня объединяют в плоскость контроля и управления).

Плоскость данных (англ. Data Plane) включает в себя сетевое оборудование: интерфейсные карты (линейные и клиентские), коммутационное оборудование, волоконно-оптическую инфраструктуру.

Плоскость управления (англ. Management Plane) совмещает функции управления сетевыми элементами EMS (англ. EMS - Element Management System) и функции управления сетью NMS (англ. NMS - Network Management System). Со стороны оператора, использующего инфраструктуру, появляется дополнительная плоскость управления - система поддержки операций/система поддержки бизнеса OSS/ BSS (англ. OSS/BSS - Operation Support System/ Business Support System).

SDN (англ. SDN - Software Defined Networking) предполагает полное отделение плоскости контроля от плоскости данных. Идеология SDN требует от плоскости управления обеспечения программируемости и автоматизации для гибкости и предоставления ресурса по запросу (слайсинга).

Плоскость контроля (англ. Control Plane), нахо дящаяся между плоскостями данных и управления, вводит интеллект в сетевые элементы, обеспечивая работу стека протоколов маршрутизации GMPLS (англ. GMPLS - Generalized Multiprotocol Label Switching). Для построения пути система управления должна "видеть" актуальную топологию сети, а значит, распространять информацию о доступности и состоянии ресурсов в системе, за это отвечает функция Resource Discovery. Сбор данных о состоянии оптических соединений и соседних сетевых элементов осуществляется в реальном времени. Функция восстановления соединений также относится к плоскости контроля.

Маршрутизация в оптической сети -более сложный механизм, чем на сетевом уровне. При формировании маршрута важно учитывать физические параметры, например потери в линии, пропускную способность на данной длине волны, оптическое отношение сигнал/шум и т.д. Как уже отмечалось, в сетях 5G для сценария URLLC (англ. - Ultra-Reliable Low Latency Network) важно оценивать задержку "из конца в конец", а значит, появляется еще один параметр для расчета пути.

\section{ПРОТОКОЛЫ МАРШРУТИЗАЦИИ И ПОИСК} ОПТИМАЛЬНОГО МАРШРУТА В ОПТИЧЕСКОЙ СЕТИ За основу GMPLS (англ. GMPLS-Generalized Multiprotocol Label Switching) принята расширенная версия протокола MPLS, разработанная группой IETF (англ. IETF - Internet Engineering Task Force), которая унаследовала принцип маркирования сообщений в соответствии с параметрами среды передачи. GMPLS рабо тает на оптическом уровне коммутации длин волн WDM (т.е. в узлах с ROADM), а также на электрическом уровне OTN, то есть в узлах с транспондерами или оборудованием кросс-коннекта (там, где выполняется разбор кадров ОTN).

Оптические соединения и узлы должны обладать собственными идентификаторами, которые необходимо предоставить плоскости управления. В качестве таких идентификаторов физических устройств (их интерфейсов) используются IP-адреса. Важно, что IP-адреса контроллеров и IP-адреса, ассоциируемые с устройствами плоскости данных, - это независимые адресные пространства.

В результате в контроллерах плоскости управления формируются таблицы IP-адресов, причем часть из них - IP-адреса контроллеров, по которым происходит обмен сигнальной информацией, а часть отображает топологию низлежащего уровня дан ных и используется для работы стека протоколов маршрутизации.

После получения каждым устройством Control Plane всей информации о топологии плоскости данных запускается алгоритм расчета известных маршру тов, а лучшие (т.е. маршруты с минимальной метрикой) помещаются в таблицу маршрутизации. В случае применения DWDM-оборудования блоки управления в каждом шасси (или сетевом узле) и образуют плоскость управления.

Маршруты обозначаются аббревиатурой LSP (англ. LSP - Label Switch Path) и представляют собой последовательность сетевых элементов, поддерживающих коммутацию (ROADM, OTN-XC, транспондеры) 
и соединяющих их оптические линии. Сетевые элементы обозначаются аббревиатурой LSR (англ. LSR - Label Switch Router).

Маршрутизация основана на графовой модели сети. Тогда LSP - это последовательность дуг (u) и вершин (v) на их стыках. Вес (или стоимость) маршрута - это сумма всех его весовых коэффициентов. Оптимальным маршрутом считается тот, весовой коэф фициент которого наименьший.

\section{Задача RWA}

Поиск оптимального маршрута в оптической сети формулируется как задача RWA (англ. RWA - Routing and Wavelength Assignment). Для построения сквозного оптического соединения важно выполнение одновременно трех условий:

- наличие непрерывного оптического маршрута для одной длины волны (Optical Path) между конечными абонентами;

- доступность ресурсов при построении данного маршрута (Resource availability);

- ухудшение качества сигнала из-за оптических эффектов в волокне не должно приводить к увеличению коэффициента ошибок выше допустимого для данного соединения.

Рекомендуемый организацией IETF для производителей оборудования оптических сетей и наиболее распространенный протокол поиска маршрута - CSPF (англ. CSPF - Constraint-based Shortest Path First) [5] на основе алгоритма Дейкстры.

В общем виде алгоритм поиска CSPF можно описать так: вычисляется "кратчайшее" соединение до следующего сетевого узла на основе метрик, сформированных оптическими ограничениями (Impairments Constraints), результат вычислений - указатель для выбора следующего узла.

Предлагается двухпараметрический алгоритм CSPF с ограничениями, характерными для 5G. Метрики рассматриваются как линейная комбинация констант различной природы: задержка (delay) и оптическое отношение сигнал/шум (OSNR). При этом коэффициенты в линейной комбинации определяются приложением "сверху" (например, в случае реализации SDN-управления), оператором сети или системой управления исходя из оптических параметров сигнала.

\section{Нахождение маршрута с помощью двухпараметрического алгоритма}

Интеллектуальная оптическая сеть должна динамически оценивать все параметры, которые, в конечном счете, оказывают влияние на качество обслуживания абонентов. Наиболее значимые параметры должны определяться конкретным приложением, запрашивающим маршрут между источником и приемником. Так, для еМВВ-сценария, в рамках которого абоненты будут обмениваться сверхширокополосным видео высокой четкости, учет задержки на Backhaul не критичен, однако важна полоса, обеспечиваемая высокоуровневым форматом модуляции, чувствительным к OSNR (англ. OSNR - Optical Signal-to-Noise Ratio) на приеме.

B URLLC-сценарии провайдер должен гарантировать минимальную задержку. Даже один лишний узел LSP с OTN-коммутацией, вносящий несколько десятков микросекунд, может ухудшить показатель качества для данного соединения.

\section{Учет задержки}

В сетевых элементах OTN-XC вносится существенно бо́льшая задержка, чем в ROADM, так как OTNкоммутация требует разбора пакетов. Значения отличаются на несколько порядков, поэтому рассмотрим худший вариант - построение маршрута на примере произвольного mesh-участка сети DWDM/OTN. Рассматриваемый участок сети изображен на рис.3, его топология соответствует сегменту Metro-core/ Backhaul согласно [3]. В качестве LSR выступают устройства с OTN кросс-коннектом в узлах A-G. 
При транзитном прохождении сигнала через узел OTN-XC будем учитывать задержки в следующих устройствах: мультиплексор и демультиплексор служебного канала OSC, мультиплексор и демультиплексор группового сигнала, фреймер с FEC, а также матрица кросс-коммутации.

Задержки в терминальных узлах (источнике и приемнике) должны быть учтены в следующем порядке: к задержкам в оптических устройствах (терминальных мультиплексорах и демультиплексорах, фильтрах служебных каналов, выравнивающих фильтрах) добавляются задержки в приемопередающих модулях. Они значительно больше задержек в устройстваX L0 и определяются фреймером и соответствующим типом FEC.

Результаты расчета задержек для транзитных узлов и оконечных узлов сети, построенной на оборудовании DWDM/OTN производства компании "T8", приведены в табл.1. Там же указаны задержки по каждому оптическому линку фрагмента сети с учетом расстояния. Отметим, что в 10-Гбит транспондерах по сравнению со 100-Гбит образцами задержка в разы больше, оборудование ОTN кросс-коннекта для высоких скоростей также вносит меньшую задержку по сравнению с 10-Гбит аналогами. Это связано в первую очередь с производительностью используемых

\section{Таблица 1. Задержки в сетевых элементах и опти-} ческих соединениях

\begin{tabular}{|c|c|c|c|}
\hline \multirow[t]{2}{*}{ Тип узла } & \multicolumn{3}{|c|}{ Задержки, мкс OTN-XC } \\
\hline & \multicolumn{2}{|l|}{100 гбит/с } & 10 гбит/с \\
\hline $\begin{array}{l}\text { Оконечный } \\
\text { узел (Тx) }\end{array}$ & \multicolumn{2}{|l|}{20,903} & 80,003 \\
\hline $\begin{array}{l}\text { Оконечный } \\
\text { узел (Rx) }\end{array}$ & \multicolumn{2}{|l|}{20,902} & 80,003 \\
\hline $\begin{array}{l}\text { Транзитный } \\
\text { узел }\end{array}$ & 5,400 & \multicolumn{2}{|r|}{24,400} \\
\hline \multicolumn{4}{|c|}{ Задержки в оптических соединениях, мкс } \\
\hline$A-B$ & 250 & $\mathrm{D}-\mathrm{B}$ & 30 \\
\hline$A-D$ & 70 & $\mathrm{D}-\mathrm{F}$ & 80 \\
\hline$A-C$ & 20 & $D-G$ & 8 \\
\hline$C-D$ & 80 & $\mathrm{~F}-\mathrm{G}$ & 30 \\
\hline $\mathrm{C}-\mathrm{E}$ & 50 & $G-B$ & 40 \\
\hline
\end{tabular}

чипов. Компонентная база высокоскоростных систем нового поколения отличается лучшими временными характеристиками.

Запишем общую формулу для задержки на выходе сетевого узла (LSR). Она складывается из задержек в оптоволокне (дуги графа, входящей в рассматриваемую вершину) и сетевом узле. Тогда метрика, определяющаяся задержкой для вершины v, может быть записана следующим образом:

$$
\mathrm{m}_{\text {delay }}(\mathrm{v})=\mathrm{m}_{\text {delay_link }}(\mathrm{v})+\mathrm{m}_{\text {delay_NE }}(\mathrm{v}) \text {. }
$$

\section{Учет оптического качества сигнала}

Основной причиной падения OSNR на сложном маршруте сегмента Backhaul можно считать шумы спонтанного усиления (англ. ASE - Amplified Spontaneous Emission) EDFA-усилителей. Для метро-агрегации и сегмента Backhaul это могут быть бустеры или предусилители, расположенные в узлах сети. На рассматриваемом фрагменте (рис.3) линейные участки, за исключением A-B, непротяженные, наличие усилителей объясняется большими потерями в пассивных элементах или ROADM.

Для наглядности и обобщения будем полагать, что каждый сетевой элемент на маршруте содержит оптический EDFA-усилитель, компенсирующий потери в предшествующем пролете и в DWDM-оборудовании.

При расчете метрики $\mathrm{m}_{\mathrm{OSNR}}$ для сетевого элемента будем учитывать шумы ASE в v, а мощность на его входе будет определяться потерями предшествующего пролета. $\mathrm{m}_{\mathrm{OSNR}}$ - это обратное значение OSNR. Тогда наилучшей дугой при формировании маршрута будет считаться та, значение которой меньше чем в остальных исходящих из вершины дугах. Обратные значения OSNR (в разах) допускают арифметическое сложение. Для расчета значения в каждой следующей вершине графа необходимо использовать в качестве слагаемого 1/OSNRin, где OSNRin - это результат расчета OSNR в предыдущей вершине (v-1). Представим формулу приращения метрики в общем виде:

$$
\mathrm{m}_{\text {OSNR }}(\mathrm{v})=\frac{1}{\mathrm{OSNR}_{\text {out }}}(\mathrm{v})=\frac{1}{\mathrm{OSNR}_{\text {in }}}+\frac{\left(\mathrm{NF}-\frac{1}{\mathrm{C}}\right) h f \Delta \mathrm{f}}{\mathrm{P}_{\text {in }}},
$$

где $\mathrm{OSNR}_{\text {in }}$ (раз) - значение оптического отношения сигнал/шум, рассчитанного для предыдущего узла, NF (раз) - коэффициент шума оптического EDFA-усилителя, G (раз) - коэффициент усиления оптического усилителя, $\mathrm{h}$ - постоянная Планка $\left(6,626 \cdot 10^{-34}\right.$ Дж·c), $\mathrm{f}($ Цц - центральная частота оптического канала, $\Delta \mathrm{f}$ - полоса оптического канала (Гц) (расчет выполняется для центральной частоты 193,9 ТГц при ширине канала 12,5 ГГц), $\mathrm{P}_{\text {in }}$ - уровень мощности 
на входе оптического усилителя в сетевом элементе с учетом потерь в оптических компонентах данного узла и входящей оптической линии.

Отметим, что поиск маршрута ведется для одноканальной системы, так как нелинейные физические эффекты, влияющие на качество сигнала, пока не учитываются. Также предполагается, что в системе достаточное число свободных длин волн, и маршрут не блокируется по причине отсутствия свободной $\lambda$ на каком-либо сегменте.

В качестве алгоритма нахождения маршрута будем использовать CSPF. Метрика представляет собой функцию, аргументами которой являются два рассмотренных выше параметра: задержка (delay) и OSNR.

$$
\mathrm{w}(\mathrm{a})=\mathrm{A} \cdot\left[\mathrm{M}_{\mathrm{OSNR}}(\mathrm{v})\right]+\mathrm{B} \cdot\left[\mathrm{M}_{\text {delay }}(\mathrm{v})\right]
$$

В формуле (3) А и В - это весовые коэффициенты, определяющие значимость OSNR и задержки соответственно. При равнозначном учете обоих слагаемых коэффициенты равны единице, если необходимость учета одного из параметров отпадает, соответствующий коэффициент обнуляется в предельном случае. $\mathrm{m}_{\mathrm{OSNR}}(\mathrm{v})$ как часть метрики определяется изменением отношения сигнал/шум на выходе сетевого элемента с учетом предшествующего линка,

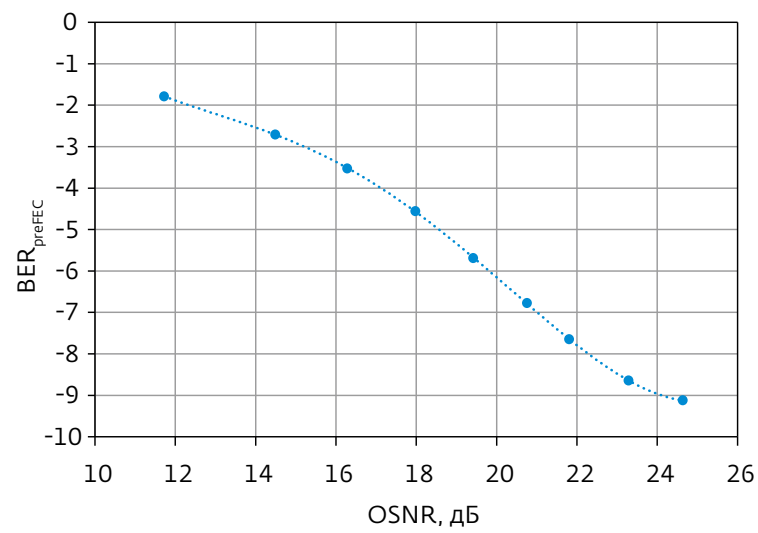

Рис.4. Зависимость коэффициента ошибок

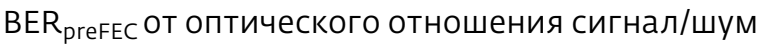
OSNR для $100 G$ транспондера, используемого в сети

$M_{\text {delay }}(v)$ - накопленной задержкой в сетевом элементе и на предшествующем линке.

Поскольку две величины вычисляются в разных единицах измерения и являются числами разных порядков, нормируем их к максимальному на сети

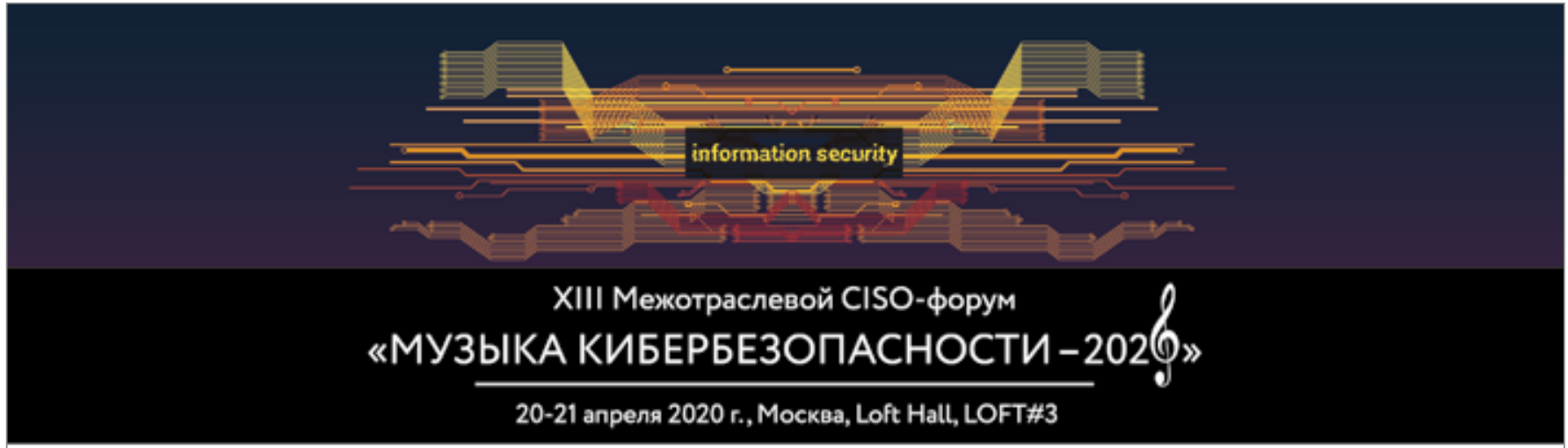

ФАКТЫ:

Только практика! Самые громкие и интересные кейсы за 2019-2020 год.

Многогранный опыт и профессиональный уровень.

А в роли жкомпозиторов» - лучшие CISO России. И музыка, много музыки!

\section{НАШИ СЕКРЕТНЫЕ ИНГРИДИЕНТЫ:}

- 500+ участников, 18 отраслей

- 70+спикеров; 80\% - CISO, которые уже успешно защищают свои компании

- TOП 10 кейсов от TOП 10 CISO: узнавай, запоминай, применяй

- 3 параллельных потока: выбирайте темы, интересные именно вам! - 5+ интерактивных дискуссий: раскроем все тайны CISO

- 4 уникальных мастер-классов от трендссеттеров: общайся и обменивайся опытом

- Увлекательная игра «Угадай мелодию»

и открытый «Караоке батл» - для закрепления дружбы фанатов легендарной CISO - музыки 
Таблица 2. Расчет составной метрики для маршрута E-C-A-B-G-D-F

\begin{tabular}{|c|c|c|c|c|c|c|c|c|c|c|}
\hline Узел & $\begin{array}{l}P_{\text {in, }} \\
\text { дБM }\end{array}$ & $\begin{array}{c}\text { OSNR } \text { out, } \\
\text { дБ }\end{array}$ & $\mathrm{m}_{\mathrm{OSNR}}(\mathrm{v})$ & $M_{\text {OSNR }}(v)$ & $\mathrm{m}_{\text {delay }}(\mathrm{v})$ & $M_{\text {delay }}(v)$ & $\begin{array}{c}M(v) \\
A=1, B=1\end{array}$ & $\begin{array}{c}M(v) \\
A=0, B=1\end{array}$ & $\begin{array}{c}M(v) \\
A=1, B=0\end{array}$ & $\begin{array}{c}M(v) \\
A \\
(S N R), B=1\end{array}$ \\
\hline$E$ & $-9,0$ & 36,69 & $5,57 \cdot 10^{-5}$ & 0,007 & 80,003 & 0,291 & 0,299 & 0,291 & 0,007 & 0,291 \\
\hline C & $-22,3$ & 28,51 & $1,19 \cdot 10^{-3}$ & 0,158 & 74,4 & 0,271 & 0,429 & 0,271 & 0,158 & 0,271 \\
\hline A & $-26,1$ & 23,7 & $2,86 \cdot 10^{-3}$ & 0,38 & 44,4 & 0,162 & 0,542 & 0,162 & 0,38 & 0,235 \\
\hline B & $-30,3$ & 19,29 & $7,51 \cdot 10^{-3}$ & 1 & 274,5 & 1 & 2 & 1 & 1 & 1,437 \\
\hline G & $-21,9$ & 18,91 & $1,09 \cdot 10^{-3}$ & 0,145 & 64,4 & 0,235 & 0,379 & 0,234 & 0,145 & 0,301 \\
\hline D & $-20,62$ & 18,64 & $8,09 \cdot 10^{-4}$ & 0,108 & 32,4 & 0,118 & 0,225 & 0,118 & 0,108 & 0,169 \\
\hline $\mathrm{F}$ & $-23,50$ & 18,17 & $1,57 \cdot 10^{-3}$ & 0,209 & 160,003 & 0,583 & 0,791 & 0,583 & 0,209 & 0,689 \\
\hline$\Sigma$ & & 18,17 & & & & & 4,66 & 2,66 & 2,006 & 3,39 \\
\hline
\end{tabular}

значению соответствующего параметра: $\max _{\mathrm{OSNR}}(\mathrm{v})$ и $\max _{\text {delay }}(\mathrm{v})$ (оцениваются заранее, до принятия решения о следующем узле маршрута). Таким образом:

$$
M_{\text {OSNR }}(\mathrm{v})=\frac{\mathrm{m}_{\mathrm{OSNR}}(\mathrm{v})}{\max _{\mathrm{OSNR}}(\mathrm{v})}, M_{\text {delay }}(\mathrm{v})=\frac{\mathrm{m}_{\text {delay }}(\mathrm{v})}{\max _{\text {delay }}(\mathrm{v})} .
$$

Определим факторы, которые могут влиять на коэф фициенты А и В в (2):

- уровень модели OSI, на котором происходит маршрутизация;

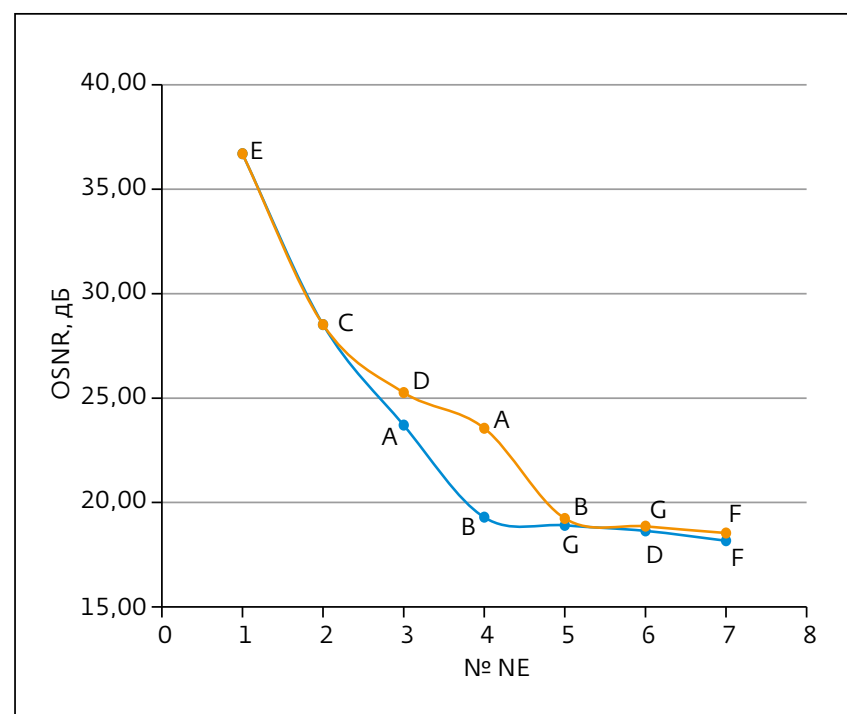

Рис.5. Деградация OSNR на двух маршрутах
- сценарий мобильного приложения;

- ручная установка коэффициентов со стороны оператора.

Наряду с перечисленными факторами необходимо упомянуть особенность транспондеров в оптических сетях, которую можно использовать при выборе значений для коэффициента А. Одна из характеристик устойчивости устройства к ошибкам - кривая транспондера. Эта функция, показывающая зависимость

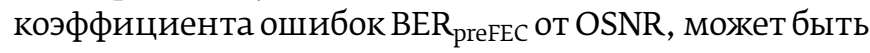
получена непосредственно от производителя или измерена лабораторно.

Приведем результат измерения кривой транспондера в режиме Back-to-back для используемых производителем "Т8" модулей (рис.4). По оси Х отложено значение OSNR, дБ, по Y - десятичный логарифм BЕR preFEс $_{\text {. }}$

Из графика видно, что при превышении определенного значения OSNR (для данного транспондера -25 дБ) обеспечивается достаточный бюджет для безошибочной передачи и нецелесообразно добиваться бо́льших значений OSNR путем оптимизации маршрута только по этому параметру. Однако у транспондера есть порог по OSNR, ниже которого ошибки за счет FEC не восстанавливаются. Для приведенного примера это значение составляет примерно 12 дБ. Отметим, что на рис.4 показан график $\mathrm{BER}_{\text {preFEc }}(\mathrm{OSNR})$, то есть до процедуры коррекции ошибок. Фактически при работе FЕС-алгоритма, если значения OSNR на входе больше 12 дБ, производитель 
Таблица 3. Расчет составной метрики для маршрута E-C-D-A-B-G-F

\begin{tabular}{|c|c|c|c|c|c|c|c|c|c|c|}
\hline Узел & $\begin{array}{l}P_{\text {in }} \\
\text { дБм }\end{array}$ & $\begin{array}{c}\text { OSNR } \\
\text { дБ }\end{array}$ & $m_{\text {OSNR }}(v)$ & $\begin{array}{c}M_{\text {OSNR }} \\
\text { (v) }\end{array}$ & $\mathrm{m}_{\text {delay }}(\mathrm{v})$ & $M_{\text {delay }}(v)$ & $\begin{array}{c}M(v) \\
A=1, B=1\end{array}$ & $\begin{array}{c}M(v) \\
A=0, B=1\end{array}$ & $\begin{array}{c}M(v) \\
A=1, B=0\end{array}$ & $\begin{array}{c}M(v) \\
A \\
(S N R), B=1\end{array}$ \\
\hline $\mathrm{E}$ & $-9,0$ & 36,69 & $5,57 \cdot 10^{-5}$ & 0,007 & 80,003 & 0,291 & 0,299 & 0,291 & 0,007 & 0,291 \\
\hline$C$ & $-22,3$ & 28,51 & $1,19 \cdot 10^{-3}$ & 0,158 & 74,4 & 0,271 & 0,429 & 0,271 & 0,158 & 0.271 \\
\hline D & $-23,5$ & 25,26 & $1,57 \cdot 10^{-3}$ & 0,209 & 104,4 & 0,380 & 0,589 & 0,380 & 0,209 & 0,405 \\
\hline A & $-23,1$ & 23,56 & $1,43 \cdot 10^{-3}$ & 0,191 & 94,4 & 0,344 & 0,534 & 0,344 & 0,191 & 0,382 \\
\hline B & $-30,3$ & 23,55 & $7,51 \cdot 10^{-3}$ & 1,0 & 274,5 & 1 & 2 & 1 & 1,0 & 1,44 \\
\hline$G$ & $-21,9$ & 19,24 & $1,09 \cdot 10^{-3}$ & 0,759 & 64,4 & 0,235 & 0,379 & 0,235 & 0,759 & 0,301 \\
\hline $\mathrm{F}$ & $-21,5$ & 18,54 & $9,90 \cdot 10^{-4}$ & 0,132 & 110,003 & 0,40 & 0,532 & 0,4 & 0,132 & 0,464 \\
\hline$\Sigma$ & & 18,54 & & & & & 4,76 & 2,92 & 1,84 & 3,56 \\
\hline
\end{tabular}

модуля гарантирует безошибочный прием благодаря применению мощных цифровых кодов.

Таким образом, в когерентных системах можно связать коэффициент А с заранее известной кривой транспондера, задав для него функцию, значения которой могут изменяться от 0 до 1 в рабочем диапазоне OSNR. Для данного модуля этот диапазон находится между 12 и 25 дБ. Чем выше по кривой

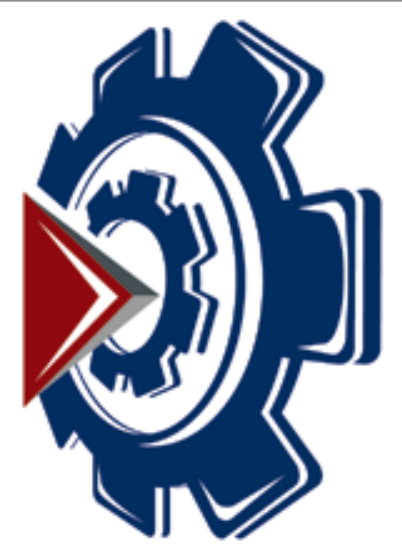

\section{ХІІІВОРОНЕЖСКИЙ ПРОМЫШЛЕННЫЙ ФОРУМ2020}

МЕЖРЕГИОНАЛЬНЫЙ ФОРУМ-ВЫСТАВКА $22-23$ а п р е л я 2020 г. ГЛАВНОЕ СОБЫТИЕ ПРОМЫШЛЕННИКОВ ЧЕРНОЗЕМЬЯ

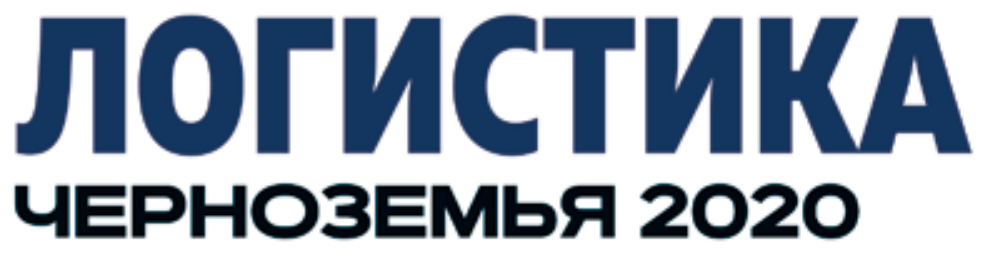

Межрегиональный форум-выставка prom@veta.ru promforum36.ru ren.+7(473) 2 100-501

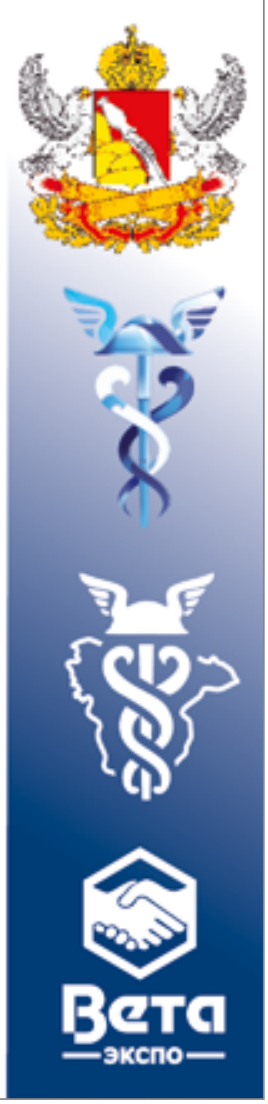


транспондера в сторону меньших OSNR по мере нарастания маршрута, тем важнее метрика OSNR, тем больше соответствующий весовой коэффициент A.

\section{Построение маршрута на сети}

Теперь сформулируем RWA-задачу. Необходимо построить маршрут на сети (рис.3) из Е в F с учетом дополнительного ограничения: трафикдолжен быть передан через все элементы сети. Выберем в качестве простых маршрутов для сравнения: E-C-A-B-GD-F и E-C-D-A-B-G-F. Ограничим поиск оптимального варианта выбором из двух LSP. Кроме того, намеренно укажем непредвиденное падение мощности на участке C-А на 5 дБ, что вызовет резкое снижение OSNR на выходе предусилителя в узле A, так как EDFA окажется в неоптимальном режиме [5]. Это сделано для наглядности, чтобы маршруты, оптимальные по OSNR и задержкам, не совпадали.

Пользуясь исходными данными о задержках (табл.1) и входных мощностях (первый столбец табл.2, 3), рассчитаем пошагово значения составных частей метрики маршрута $\left(\mathrm{m}_{\mathrm{OSNR}}(\mathrm{v}), \mathrm{m}_{\text {delay }}(\mathrm{v})\right)$ и их нормированных значений $\left(\mathrm{m}_{\mathrm{OSNR}}(\mathrm{v}), \mathrm{M}_{\text {delay }}(\mathrm{v})\right)$. Результаты расчета приведены в табл. 2 и 3.

По третьему столбцу таблицы можно проследить деградацию OSNR на каждом LSR. Красным в таблицах выделены значения OSNR в той области кривой

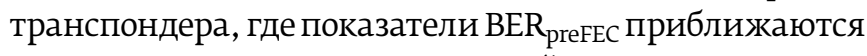
к границе допустимых значений. Для наглядности построим соответствующие графики зависимости OSNR (дБ) от номера сетевого элемента (NE) (рис.5).

В последних четырех столбцах указаны значения двухпараметрической метрики на каждом шаге, а в заключительной строке приведено ее результирующее значение в финальной точке маршрута при раз личных комбинациях коэффициентов А и В.

Как уже отмечалось, коэффициенты могут быть функциями, а не константами. В частности, коэффициент А может зависеть от точки кривой транспондера (рис.4). На основе функции $\mathrm{BER}_{\text {preFEc }}(\mathrm{OSNR})$ ана литически определены значения коэффициента A в разных точках маршрута, а метрика маршрута рассчитывалась пошагово с учетом динамического весового коэффициента. Результаты сведены в последних столбцах табл.2 и 3.

\section{Анализ результатов}

1. При учете задержек и изменений $\operatorname{OSNR}(A=1, B=1)$ оптимальным является маршрут E-C-A-B-G-D-F, поскольку его суммарный вес незначительно меньше $(M(v)=4,66$ и $M(v)=4,67$ соответственно).

2. При учете только задержек $(A=0, B=1)$ в качестве оптимального выбран маршрут E-C-A-B-G-D-F, который существенно превосходит первый вариант $(M(v)=2,66$ и $M(v)=2,92$ соответственно).

3. Если важен только OSNR $(A=1, B=0)$, выигрывает маршрут E-C-D-A-B-G-F, который отличается меньшим суммарным весом $(\mathrm{M}(\mathrm{v})=2,0$ и $\mathrm{M}(\mathrm{v})=1,84$ соответственно).

4. При расчете метрики маршрутов с динамическим коэффициентом A $\left(\mathrm{A}=\mathrm{F}\left(\mathrm{BER}_{\text {preFEc }}(\mathrm{OSNR})\right)\right.$ и постоянным коэффициентом $\mathrm{B}=1$ оптимальным считается маршрут E-C-A-B-G-D-F (M(v)=3,39 и $\mathrm{M}(\mathrm{v})=3,56$ соответственно).

\section{Выводы}

В статье показано, каким образом при формировании метрики маршрута можно одновременно учитывать параметры разной природы и рассчитывать оптимальный маршрут в сегменте Backhaul. На примере фрагмента Backhaul, реализованного на OTN/DWDMоборудовании с поддержкой стека протоколов GMPLS, представлен алгоритм поиска маршрута для установ ления сквозного соединения по запросу от сервиса 5G.

Соединение может устанавливаться по критерию минимальной задержки или максималь ной пропускной способности, требующей высокого OSNR на приеме. Метрика маршрута - это линейная комбинация, учитывающая с соответ ствующими сервису коэффициентами изменение задержки или оптического отношения сигнал/шум. Как показал расчет, оптимальные маршруты могут отличаться в зависимости от выбранных весовых коэффициентов.

Реализация предложенного алгоритма маршрутизации в оптической сети OTN/DWDM требует взаимодействия всех логических плоскостей: дан ${ }^{-}$ ных, контроля, управления. Только так можно обеспечить предоставление пропускной способности по требованию и быструю реконфигурацию транс портных ресурсов с учетом сценария IMT-2020/5G.

\section{ЛИТЕРАТУРА}

1. Богданова Е. Транспортная сеть 5G/IMT-2020 // ПЕРВАЯ МИЛЯ. 2019. № 7. С. 40-47.

2. FiberHome, China Telecommunications. Lite and Flexible OTN for next generation (5G) Mobile Fronthaul Transport // SG15 - C.0064. - Geneva, 2017.

3. Supplement 67 to ITU-T G-series Recommendations. Application of optical transport network Recommendations (07/2019).

4. Mahi Networks, Inc. Implementing a CSPF Algorithm in Intelligent Optical Networks. 2003.

5. Толстихин И., Богданова Е., Сигаев А. Имитационнное моделирование протяженных DWDMсистем // ПЕРВАЯ МИЛЯ. 2016. № 4. С. 50-55. 
